\title{
The Convex Hull of the Transposition Matrices
}

\author{
Lambert S. Joel \\ Institute for Basic Standards, National Bureau of Standards, Washington, D.C. 20234
}

(June 26, 1974)

\begin{abstract}
The convex hull of the $n$ by $n$ transposition matrices is characterized as the set of symmetric doubly stochastic matrices with trace $n-2$. A similar characterization (with trace $\geqslant n-2$ ) is given for the convex hull of the union of transposition matrices and the identity matrix.
\end{abstract}

Key words: Combinatorial analysis; convex set; linear inequalitites; permutations.

Many combinatorial optimization problems can be expressed as requiring the extremization of a linear function over some finite set $S$ of points in a real $N$-dimensional space. To bring the theoretical and computational resources of linear programming to bear, it is necessary to characterize the convex hull $K(S)$ of $S$ as the solution-set of a "nicely structured" family of linear inequalities and equations.

The outstanding example, arising in connection with the assignment problem of operations research, has $S=S_{n}$, the set of $n$ by $n$ permutation matrices (regarded as points in $n^{2}$ dimensionat space). Here a well-known theorem ${ }^{1}$ (Birkhoff-Hoffman-von Neumann-Wielandt et al.) identifies $K\left(S_{n}\right)$ as the set of all $n$ by $n$ doubly stochastic matrices $X=\left(x_{i j}\right)$, i.e. matrices with nonnegative entries and with each row and column summing to 1 . It is expected that a similar characterization of $K\left(C_{n}\right)$, where $C_{n}$ is the set of all cyclic permutation matrices, would be valuable in connection with the traveling saleman problem, but no such characterization has been given as yet.

For a given $n$ let $\Pi_{c}(1 \leqslant c \leqslant n)$ denote the set of $n$ by $n$ permutation matrices for which the decomposition of the associated permutation into disjoint cycles contains exactly $c$ cycles (including cycles of length one). Since $C_{n}=\Pi_{1}$, the remark ending the last paragraph suggests looking at the "other end" of the sequence $\left\{\Pi_{c}\right\}_{c=1}^{n}$. The situations for $\Pi_{n}$ and $\Pi_{n-1}$ are simple, and form the subject of this note. Clearly $\Pi_{n}$ consists of the identity matrix $I_{n}$, so that $K\left(\Pi_{n}\right)=\left\{I_{n}\right\}$. We go on to characterize $K\left(\Pi_{n-1}\right)$ as well as $K\left(\Pi_{n-1} \cup \Pi_{n}\right)=K\left(\Pi_{n-1} \cup\left\{I_{n}\right\}\right)$. Note that $\Pi_{n-1}$ consists of the $n(n-1) / 2$ transposition matrices $T_{p q}(1 \leqslant p<q \leqslant n)$ defined by

$$
\begin{array}{ll}
\left(T_{p q}\right)_{i j}=1 & \text { if }(i, j)=(p, q) \text { or }(q, p), \\
\left(T_{p q}\right)_{i i}=1 & \text { for } i \neq p, q, \\
\left(T_{p q}\right)_{i j}=0 & \text { otherwise. }
\end{array}
$$

THEOREM 1. $\mathrm{K}\left(\Pi_{\mathrm{n}-1}\right)$ consists of all symmetric doubly stochastic matrices with trace $\mathrm{n}-2$.

THEOREM 2. $\left.\mathrm{K}\left(\Pi_{\mathrm{n}-1}\right) \cup\left\{\mathrm{I}_{\mathrm{n}}\right\}\right)$ consists of all symmetric doubly stochastic matrices with trace $\geqslant \mathrm{n}-2$.

To begin the proof, note that each member of $\Pi_{n-1}$ (of $\Pi_{n-1} \cup\left\{I_{n}\right\}$ ) is a symmetric doubly stochastic matrix with trace $n-2$ (with trace $\geqslant n-2$ ). It readily follows that the same is true for each member of $K\left(\Pi_{n-1}\right)$ (of $\left.K\left(\Pi_{n-1} \cup\left\{I_{n}\right\}\right)\right)$.

AMS Subject Classification: 05B20, 1 ๖A51.

${ }^{1}$ Cf. Chap. 5: H. J. Ryser, Combinatorial Mathematics, Carus Mathematical Monograph No. 14, MAA, John Wiley \& Sons 1963. 
Next, let $X=\left(x_{i j}\right)$ be any symmetric doubly stochastic matrix. Note that

$$
\sum_{p} \sum_{q>p} X_{p q}=\frac{1}{2} \sum_{p, q \neq p} X_{p q}=\frac{1}{2}[n-\operatorname{tr}(X)] .
$$

The matrix

$$
Y=X-\sum_{p} \sum_{q>p} x_{p q} T_{p q}
$$

is readily seen to be diagonal, with $(i, i)$ entry

$$
\begin{gathered}
y_{i i}=x_{i i}-\Sigma\left\{x_{p q}: p \neq i, q \neq i, q>p\right\} \\
=x_{i i}-\sum_{p<i}\left(\sum_{q>p}\left(x_{p q}-x_{p i}\right)\right)-\sum_{p>i} \sum_{q>p} x_{p q} \\
=x_{i i}+\sum_{p<i} x_{p i}-\sum_{p \neq i} \sum_{q>p} x_{p q} \\
=\sum_{p \leq i} x_{p i}-\left[\sum_{p} \sum_{q>p} X_{p q}-\sum_{q>i} X_{i q}\right] \\
=\left[\sum_{p \neq i} x_{p i}+\sum_{q>i} x_{q i}\right]-\sum_{p} \sum_{q>p} x_{p q} .
\end{gathered}
$$

Since column $i$ of $X$ sums to l, the last result together with (1) yields

$$
y_{i i}=1-\frac{1}{2}[n-\operatorname{tr}(X)]=\frac{1}{2}[\operatorname{tr}(X)-(n-2)]=\delta,
$$

where the final equation defines $\delta$.

If $\operatorname{tr}(X)=n-2$, then (2) yields $Y=0$ and (1) shows that $X=\Sigma x_{p q} T_{p q}$ lies in $K\left(\Pi_{n-1}\right)$, completing the proof of Theorem 1. If $\operatorname{tr}(X) \geqslant n-2$, then $\delta \geqslant 0$; since (2) shows that $Y=\delta I_{n}$ and since (1) and (2) yield $\Sigma x_{p q}+\delta=1$, it follows that $X=\Sigma x_{p q} T_{p q}+\delta I_{n}$ lies in $K\left(\Pi_{n-1} \cup\left\{I_{n}\right\}\right)$, completing the proof of Theorem 2.

(Paper 78B3-409) 